\title{
The protective role of pregnane $X$ receptor in lipopolysaccharide/D-galactosamine-induced acute liver injury
}

\author{
Kun Wang ${ }^{1}$, Ivan Damjanov ${ }^{2}$ and Yu-Jui Yvonne Wan ${ }^{1}$
}

The pregnane $\mathrm{X}$ receptor $(\mathrm{PXR})$ is a nuclear receptor transcription factor regulating drug-metabolizing enzymes and transporters that facilitate xenobiotic and endobiotic detoxification. Recent studies show that PXR is important in abrogating intestinal tissue damage. This study examines the role of PXR in lipopolysaccharide (LPS)/D-galactosamine (GaIN)-induced acute liver injury using wild-type and PXR-null mice. LPS/GalN-treated PXR-null mice had greater increases of alanine transaminase (ALT), hepatocyte apoptosis, necrosis, and hemorrhagic liver injury than wild-type mice.

LPS/GalN-mediated phosphorylation of JNK $1 / 2$ and ERK1/2 was differentially regulated in wild-type and PXR-null mice. Importantly, LPS/GalN-induced hepatic Stat3 survival signaling was impaired and early activation of Jak2 was delayed in PXR-null mice. Expression levels of pro-survival proteins Bcl-xL and heme oxygenase-1 (HO-1), which are downstream of Stat3, were substantially lower in PXR-null than wild-type mouse livers after LPS/GalN treatment. Autophagy is also involved in LPS/GalN-induced liver injury. Lack of PXR resulted in a significant reduction of LC3B-I, -II as well as Beclin-1 protein levels after LPS/GalN treatment. In addition, PXR is implicated in hepatocytes homeostasis. Taken together, PXR is a critical hepatoprotective factor. Increases of LPS/GalN-induced hepatocyte apoptosis and liver injury in PXR-null mice are due to deregulated mitogen-activated protein (MAP) kinase activation as well as delayed Jak2/Stat3 activation, which lead to a compromise in defense mechanisms that involve Bcl-xL-, HO-1, and autophagy-mediated pathways.

Laboratory Investigation (2010) 90, 257-265; doi:10.1038/labinvest.2009.129; published online 7 December 2009

KEYWORDS: apoptosis; Jak2/Stat3; liver injury; MAP kinases; PXR

The binding of TNF $\alpha$ to TNFR1 induces rapid activation of several intracellular signaling pathways that influence cell survival and death. ${ }^{1}$ Among them, nuclear factor- $\kappa \mathrm{B}$ (NF$\kappa \mathrm{B})$ is the major transcription factor that regulates antiapoptotic proteins and prevents cells from TNF $\alpha$-induced apoptosis. ${ }^{1,2}$ Deletion of p65RelA, a key player of NF- $\kappa$ B signaling, causes severe hepatocyte apoptosis and embryonic lethality due to defective NF- $\kappa \mathrm{B}$ signaling and uncontrolled TNF $\alpha$ toxicity. ${ }^{3}$ The Stat 3 transcription factor also has an essential role in cell growth and apoptosis. Mice with specifically blocked hepatocyte Stat 3 activation have increased apoptosis and liver injury induced by lipopolysaccharide (LPS), suggesting that Stat3-mediated survival signaling protects hepatocytes from $\mathrm{TNF} \alpha$-induced apoptosis. ${ }^{4}$ In addition, activation of Stat3 blocks toll-like receptor-mediated inflammation. ${ }^{5}$ TNF $\alpha$ can induce mitogen-activated protein kinases (MAP kinases), including JNK1/2, ERK1/2, and p38, which transmit extracellular signals into their intracellular targets to regulate cell proliferation, differentiation, and apoptosis. ${ }^{1}$ Activation of MAP kinases has dual roles and presents both pro-apoptotic and anti-apoptotic functions in TNF $\alpha$-mediated cell death events. JNK knockout mice are resistant to $\mathrm{TNF} \alpha$-induced liver injury, indicating that JNK mediates cell death signaling and promotes liver injury. ${ }^{6}$ In general, ERK signaling has been considered to mediate cell survival; however, increasing evidence shows that ERK pathway also mediates apoptosis and tissue damage induced by different stimuli in different tissues. ${ }^{7-9}$ Depending

\footnotetext{
${ }^{1}$ Department of Pharmacology, Toxicology and Therapeutics, University of Kansas Medical Center, Kansas City, KS, USA and ${ }^{2}$ Department of Pathology and Laboratory Medicine, University of Kansas School of Medicine, Kansas City, KS, USA

Correspondence: Dr Y-JY Wan, PhD, Department of Pharmacology, Toxicology and Therapeutics, University of Kansas Medical Center, 3901 Rainbow Boulevard, Kansas City, KS 66160, USA.

E-mail: ywan@kumc.edu

Received 10 July 2009; revised 4 September 2009; accepted 16 September 2009
} 
on cell type or model of damage, inhibition of p38 can enhance ${ }^{10}$ or prevent ${ }^{11} \mathrm{TNF} \alpha$-induced apoptosis, thus the role of p38 in $\mathrm{TNF} \alpha$-induced cell or tissue injury remains controversial.

Nuclear receptor pregnane $\mathrm{X}$ receptor (PXR) regulates a range of genes associated with clearance of endobiotics and xenobiotics. Recent research has revealed the important role of PXR in intestine injury and inflammatory processes. ${ }^{12-14}$ Crosstalk between PXR and NF- $\kappa \mathrm{B}$ has been reported. ${ }^{12}$ Using in vitro models, it has been implicated that activated human PXR (hPXR) suppresses NF- $\kappa \mathrm{B}$ and its target genes in primary human hepatocytes and intestinal LS180 cells. ${ }^{12}$ Activation of rodent PXR ameliorates dextran sulfate sodium-induced colitis through repression of NF- $\kappa \mathrm{B}$ target genes. ${ }^{13}$ Notably, activated PXR also directly up-regulates anti-apoptotic proteins such as Bcl-xL and Bcl-2 in cultured hepatocytes ${ }^{15}$ and colon cancer cells. ${ }^{16}$ Activation of PXR inhibits human hepatic stellate cell trans-differentiation in vitro ${ }^{17}$ and in carbon tetrachloride-induced liver fibrosis mouse model. ${ }^{18}$ PXR also provides neuroprotection in a mouse model of Niemann-Pick C disease. ${ }^{19}$

Despite emerging reports about the regulatory role of PXR in many physiological and pathological conditions, little is known about whether PXR regulates acute hepatic injury in vivo and whether survival or pro-apoptotic signaling pathways are affected due to lack of PXR. LPS/D-galactosamine (GalN) treatment of mice is a classic model of acute liver injury. GalN is a specific hepatotoxic transcriptional inhibitor that abolishes protection of NF- $\kappa \mathrm{B}$ and sensitizes liver toward subtoxic amounts of LPS, ${ }^{20}$ and TNF $\alpha$ is the predominant mediator that induces hepatocyte apoptosis and liver injury. ${ }^{21,22}$ Our study examines the in vivo role of PXR in LPS/GalN-induced acute liver injury using wild-type and PXR-null mice. We specifically determined the in vivo impact of PXR on LPS/GalN-induced activation of hepatic MAP kinases and Stat3 as well as the downstream survival and death pathways. Our results are the first to show that loss of PXR potentiates LPS/GalN-induced hepatocyte apoptosis and liver injury. The enhanced liver injury found in PXR-null mice is associated with delayed activation of hepatic Stat3, deregulated phosphorylaton of MAP kinases, and a compromise in anti-apoptotic signaling.

\section{MATERIALS AND METHODS \\ Reagents}

Antibodies specific for phospho-Akt (Thr308), pan-Akt; phospho-Stat3 (Tyr705), phospho-Stat3 (Ser727), Stat3; phospho-SAPK/JNK (Thr183/Tyr185), SAPK/JNK; phosphop38 MAP kinase (Thr180/Tyr182), p38 MAP kinase; phospho-ERK1/2 MAP Kinase (Thr202/Tyr204), ERK1/2 MAP Kinase; phospho-Jak1 (Tyr1022/1023); phospho-Tyk2 (Tyr 1054/1055); phospho-Jak2 (Tyr1007/1008), Jak2; cleaved caspase-3 (Asp175), Bcl-xL and Bax were purchased from Cell Signaling Technology. Antibody specific for phosphorp65 (Ser311), heme oxygenase-1 (HO-1) were purchased from Santa Cruz Biotechnology Inc. Antibody for Gapdh was purchased from Abcam. LPS from Escherichia coli 0127: B8 and GalN were purchased from Sigma-Aldrich.

\section{Experimental Design and Biochemical Analysis}

Wild-type and PXR-null mice with a mixed background of C57B and SVJ129 were kindly provided by Dr Wen Xie at University of Pittsburgh and the generation of PXR-null mice were described earlier. ${ }^{23,24}$ All procedures were conducted in accordance with the National Institutes of Health Guidelines for the Care and Use of Laboratory Animals and were approved by the Kansas University Medical Center Institutional Animal Care and Use Committee. Male mice (9-11 weeks, $n=5$ for each treatment group or time point) were administered with one intra-peritoneal (i.p.) dose of $100 \mu \mathrm{g} / \mathrm{kg}$ of LPS and $700 \mathrm{mg} / \mathrm{kg}$ of GalN dissolved in sterile $0.9 \%$ saline, control mice were injected with sterile $0.9 \%$ saline. Livers were removed at 0,1 , and $5 \mathrm{~h}$ after LPS/GalN injection. Stock LPS was dissolved in sterilized saline at $1 \mathrm{mg} / \mathrm{ml}$ and sonicated for $5 \mathrm{~min}$, aliquot and stored at $-80^{\circ} \mathrm{C}$. LPS and GalN were freshly prepared before usage. Serum alanine transaminase (ALT) levels were monitored using the Liquid ALT Reagent kit (Pointe Scientific Inc.).

\section{Hematoxylin-eosin Staining and TUNEL Assay of Liver Sections}

Liver tissues were fixed with $10 \%$ buffered formalin/PBS, embedded in paraffin. Liver sections $(5 \mu \mathrm{m})$ were stained with hematoxylin and eosin (H\&E) following standard protocol. TUNEL assay was performed using the In situ Cell Death Detection kit (Roche Molecular Biochemicals).

\section{Western Blot}

Liver proteins (40-100 $\mu \mathrm{g}$ ) were separated on sodium dodecyl sulfate-polyacrylamide gel (10-15\%) and then electro-transferred to PVDF membranes. Membranes were incubated with primary antibody overnight at $4{ }^{\circ} \mathrm{C}$, followed by incubation with secondary antibody and detected by enhanced chemical luminescence kit. Membranes were re-probed with antiGapdh antibody to monitor equal loading of protein. Representative blots are shown in the figures. Densitometry analysis was performed to quantify protein bands.

\section{Statistical Analysis}

The results (ALT levels) were analyzed using Student's $t$ test. All data are expressed as mean \pm s.d. $P<0.05$ was considered significant.

\section{RESULTS \\ LPS/GaIN-induced Liver Injury is Enhanced due to Lack of PXR}

Five hours after LPS/GalN injection, PXR-null mice had higher serum ALT (about $1100 \mathrm{IU} / \mathrm{l}$ ) than that of wild-type mice (about $150 \mathrm{IU} / \mathrm{l}$ ) (Figure 1a). LPS/GalN treatment 


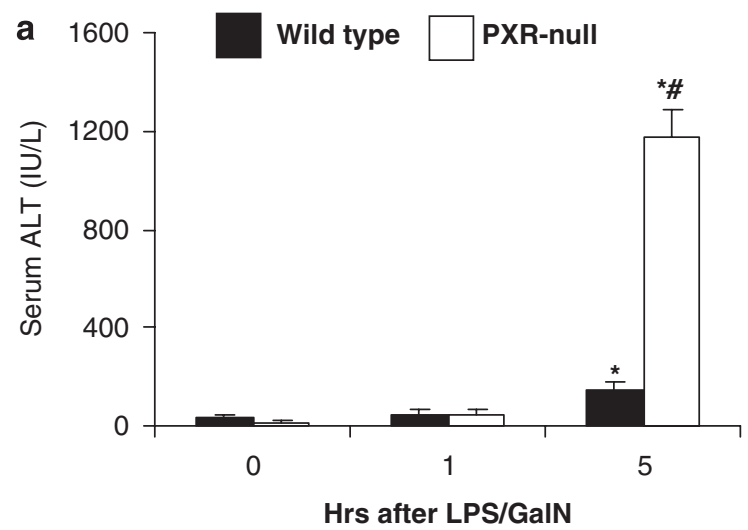

b
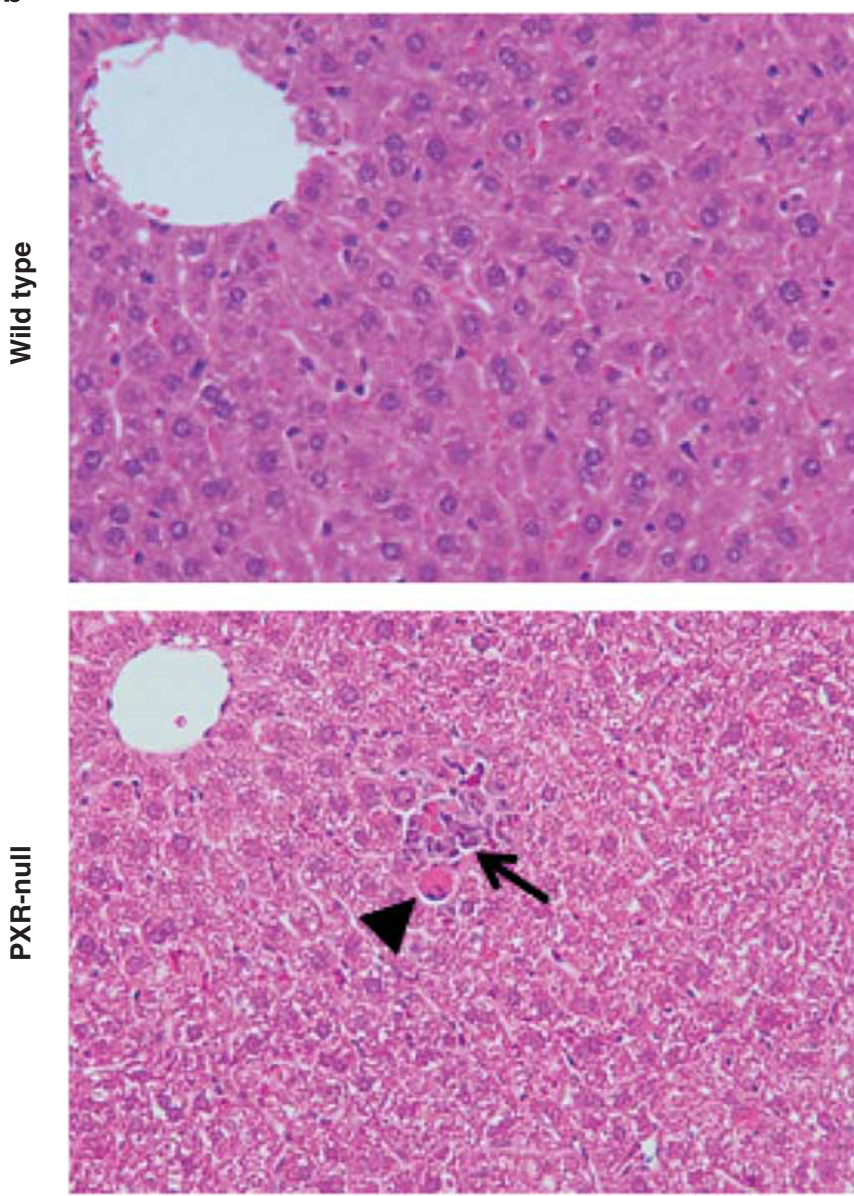

LPS/GaIN
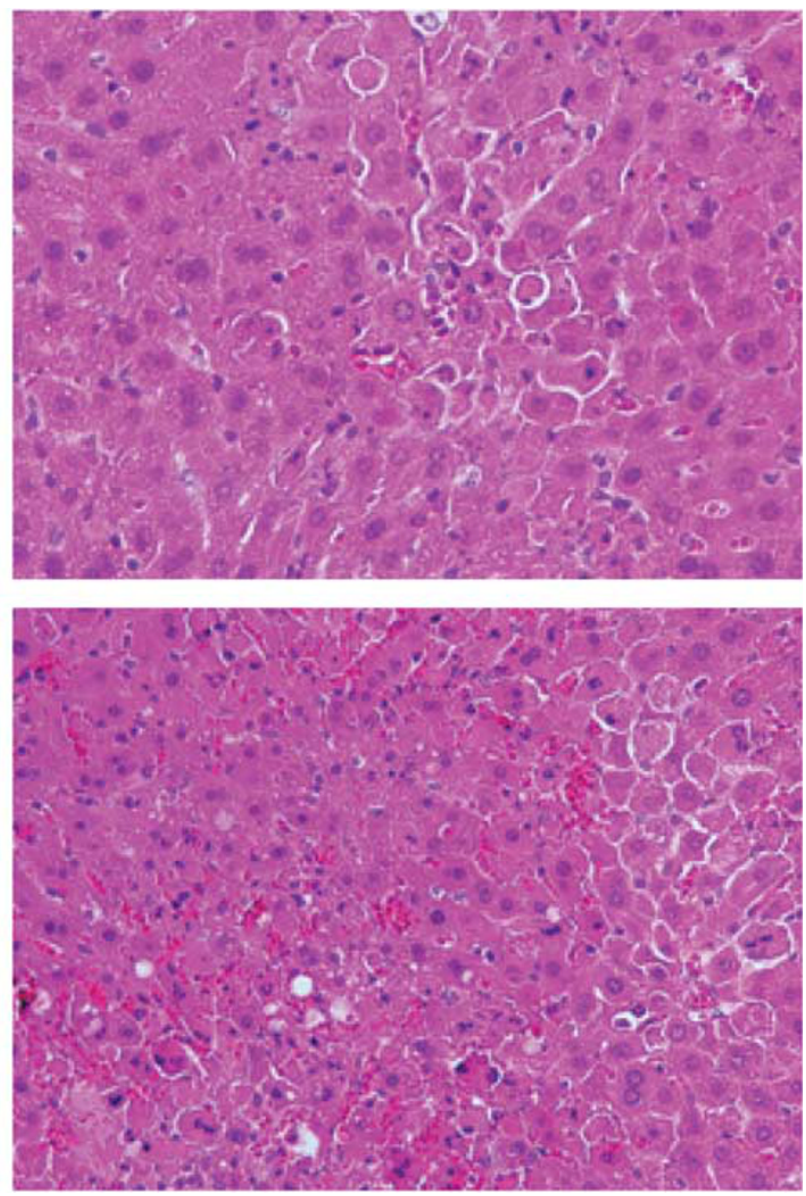

Figure 1 PXR-null mice are sensitive to LPS/GalN-induced liver injury. Mice were i.p. injected with LPS (100 $\mu \mathrm{g} / \mathrm{kg})$ and GalN (700 mg/kg) or saline. (a) ALT levels. Data are represented as means \pm s.d. ( $n=5 /$ group/time point). (b) Representative H\&E-stained liver sections of wild-type and PXR-null mice $5 \mathrm{~h}$ after LPS/GalN injection. Sporadically, apoptotic hepatocytes (triangle) and neutrophil infiltrations (arrow) were observed in liver sections of saline-treated PXRnull mice. ${ }^{* P}<0.05$, compared with wild-type mice of $0 \mathrm{~h}$; ${ }^{\#} P<0.05$, compared within same time point.

caused apoptotic, focal necrotic, and hemorrhagic injury in the livers of PXR-null mice; in contrast, apoptosis and necrosis was much less in the livers of LPS/GalN-treated wild-type mice. Importantly, no massive hemorrhage was present in the livers of wild-type mice (Figure 1b). Thus, in the absence of PXR, LPS/GalN-induced liver injury is potentiated. Sporadic apoptotic hepatocytes and neutrophil infiltrations were occasionally present in the liver sections of saline-treated PXR-null mice (Figure 1b, arrow and triangle), although livers of saline-treated wild-type mice had normal 


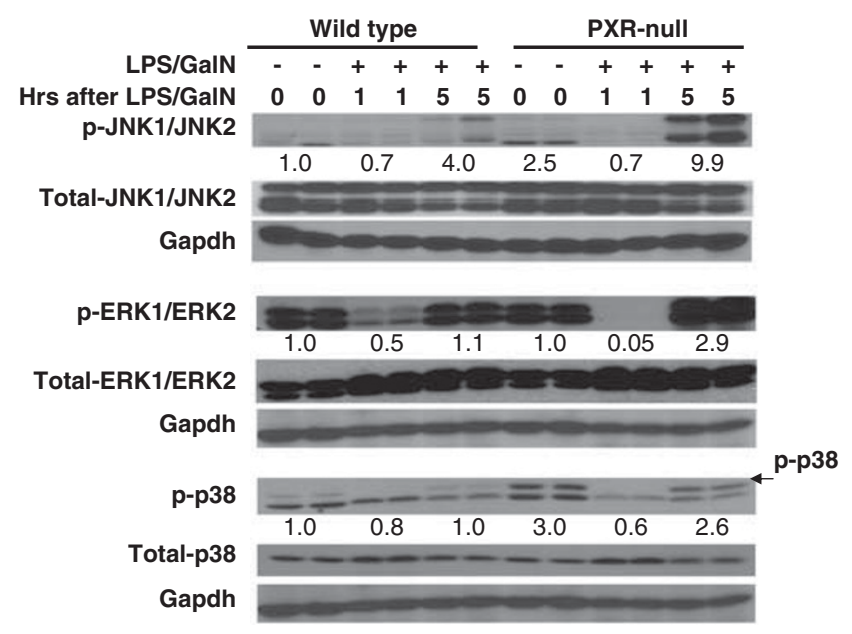

Figure 2 Phosphorylation of hepatic MAP kinases is differentially altered by LPS/GalN in wild-type and PXR-null mice. Mice were i.p. injected with LPS $(100 \mu \mathrm{g} / \mathrm{kg})$ and GaIN $(700 \mathrm{mg} / \mathrm{kg})$ or saline ( $n=5 / \mathrm{group} /$ time point). Livers were removed at the time point as indicated. Liver proteins were immunoblotted with indicated antibodies and representative samples are shown in the figure.

morphology (Figure 1b). These findings suggest PXR provides hepato-protective effects.

\section{LPS/GaIN-mediated Phosphorylation of MAP Kinases is Altered due to Lack of PXR}

Five hours after LPS/GalN injection, phosphorylation of hepatic JNK1/2 (Thr183/Tyr185) (p-JNK1/2) was much higher in PXR-null than wild-type mice (Figure 2). One hour after LPS/GalN injections, levels of p-ERK1/2 (Thr202/ Tyr204) were substantially reduced in both genotypes; however, trace levels of hepatic p-ERK1/2 were still detected in wild-type mice, but not in PXR-null mice (Figure 2). Five hours after treatment, levels of p-ERK1/2 were higher in PXR-null than wild-type mice. PXR-null mice had increased basal levels of p-p38 (Thr180/Tyr182) (Figure 2). Similar to the expression pattern of ERK1/2, LPS/GalN treatment transiently reduced p-p38 within $1 \mathrm{~h}$. At $5 \mathrm{~h}$, levels of p-p38 were slightly higher in PXR-null than wild-type mice. Thus, rapid and substantial deactivation of MAP kinases at early time point $(1 \mathrm{~h})$ and elevated activation of MAP kinases at late time point $(5 \mathrm{~h})$ were associated with increased and accelerated liver injury in PXR-null mice.

\section{Phosphorylation of Hepatic Stat3 Induced by LPS/GaIN is Delayed due to Lack of PXR}

One hour after LPS/GalN injection, p-Stat3 (Tyr705) was present only in wild-type mice (Figure $3 \mathrm{a}$ ). In contrast, p-Stat3 (Tyr705) was detected in PXR-null mice $5 \mathrm{~h}$ after injection (Figure 3a). In addition, LPS/GalN induced similar levels of p-Stat 3 at Ser727 in wild-type and PXR-null mice (Figure 3a). As Stat3 mediates survival signaling in LPS/ GalN-induced liver injury, the impaired activation of hepatic

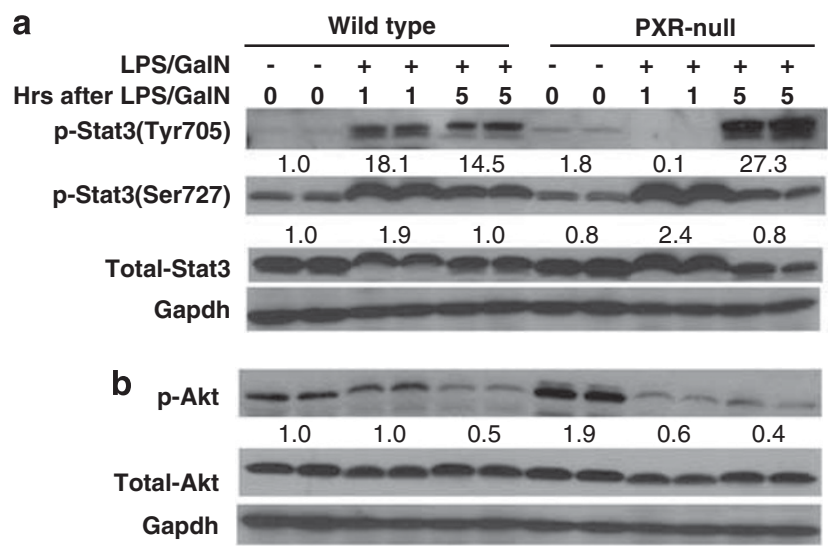

Figure 3 Activation of Stat3 by LPS/GalN is delayed in the livers of PXRnull mice. (a) Stat 3 protein data. (b) Akt protein data. Mice were i.p. injected with LPS $(100 \mu \mathrm{g} / \mathrm{kg})$ and GalN $(700 \mathrm{mg} / \mathrm{kg})$ or saline $(n=5 / \mathrm{group} /$ time point). Livers were removed at the time point as indicated. Liver proteins were immunoblotted with indicated antibodies and representative samples are shown in the figure.

Stat3 at Tyr705 might account for the increased liver injury in PXR-null mice.

Activation of Akt provides protective effects against TNF $\alpha$ mediated apoptosis in endothelial cells, ${ }^{25}$ renal cells, ${ }^{26}$ and liver injury model. ${ }^{27}$ As shown in Figure 3b, PXR-null mice had slightly increased basal p-Akt (Thr308), which was similar to the pattern of $\mathrm{p} 38$. LPS/GalN reduced the levels of p-Akt in wild-type and PXR-null mice, and the reduction seems more pronounced in PXR-null than wild-type mice (Figure $3 b$ ).

\section{LPS/GaIN-induced Activation of Upstream Kinases of Stat3 is Altered in Wild-type and PXR-null Mice}

Stat 3 is selectively phosphorylated by the upstream Janus protein kinase (Jak) family, which includes Jak1-3 and Tyk2. Jak1, Jak2, and Tyk2 are widely expressed, whereas Jak3 is primarily found in lymphoid and myeloid cells. ${ }^{28}$ As p-Stat3 (Tyr705) was delayed in LPS/GalN-treated PXR-null mice, we examined whether lack of PXR affects activation of kinases upstream of Stat3. One hour after LPS/GalN injection, increased hepatic p-Jak2 (Tyr1007/1008) occurred only in wild type, but not in PXR-null mice; at $5 \mathrm{~h}$, wild-type and PXR-null mice had similar levels of p-Jak2 (Figure 4). LPS/GalN did not induce hepatic p-Jak1 (Tyr1022/1023) until $5 \mathrm{~h}$ after LPS/GalN injection in both genotypes of mice, and the induction was weak. Therefore, Jak2, but not Jak1, is mostly associated with LSP/GalN-mediated Stat3 activation. In addition, LPS/GalN did not change the level of hepatic p-Tyk2 (Tyr1054/1055) in wild-type and PXR-null mice at $1 \mathrm{~h}$. At $5 \mathrm{~h}$, the levels of p-Tyk2 were slightly reduced in PXRnull mice. Thus, loss of PXR primarily affects LPS/GalNinduced p-Jak2 at $1 \mathrm{~h}$ (Figure 4). The data indicate that the impaired activation of Jak2 accounts for the delayed activation of Stat3 in PXR-null mice. 


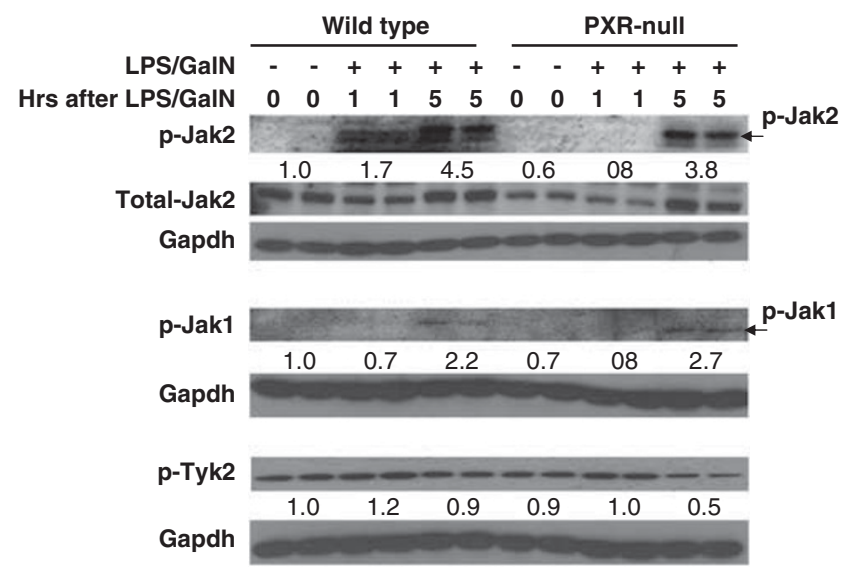

Figure 4 Loss of PXR impairs LPS/GalN-induced activation of Jak2 in the livers of PXR-null mice. Mice were i.p. injected with LPS $(100 \mu \mathrm{g} / \mathrm{kg})$ and GalN $(700 \mathrm{mg} / \mathrm{kg}$ ) or saline ( $n=5 / \mathrm{group} /$ time point). Livers were removed at the time point as indicated. Hepatic cytosal fractions were immunoblotted with indicated antibodies. Representative samples are shown in the figure.

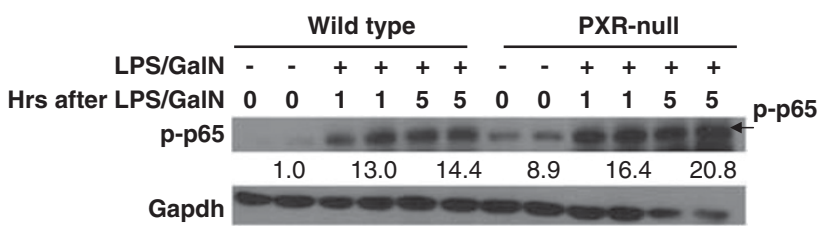

Figure $5 \mathrm{LPS} / \mathrm{GalN}$-induced activation of p65 is augmented in the livers of PXR-null mice. Mice were i.p. injected with LPS $(100 \mu \mathrm{g} / \mathrm{kg})$ and GalN (700 mg $/ \mathrm{kg}$ ) or saline ( $n=5 / \mathrm{group} /$ time point). Livers were removed at the time point as indicated. Liver proteins were immunoblotted with indicated antibody and representative samples are shown in the figure.

\section{LPS/GalN-induced Activation of NF- $\kappa$ B is Augmented in PXR-null Mice}

We performed western blot analysis using antibody specific to phosphorylated p65 (p-p65). The basal p-p65 increased due to lack of PXR (Figure 5). LPS/GalN further increased activation of p-p65, and the levels of p-p65 were much higher in PXR-null than wild-type mice (Figure 5). The highest levels of p-p65 were observed in the livers of PXR-null mice at $5 \mathrm{~h}$, which was correlated with activation of Stat 3 at the same time point in PXR-null mice. Our results indicate that in the absence of PXR, activation of p65 is enhanced, which may cause deregulated TNF $\alpha$ and/or IL- 6 release as well as Stat 3 activation.

\section{LPS/GaIN-induced Hepatocyte Apoptosis is Augmented in PXR-null Mice}

LPS/GalN-induced hepatocyte apoptosis was examined by TUNEL staining. Five hours after LPS/GalN injection, PXRnull mice had three times more TUNEL-positive hepatocytes than wild-type mice did (Figure 6a and b). One hour after LPS/GalN injection, cleaved caspase-3 was not detected in the livers of wild-type and PXR-null mice (data not shown). At
$5 \mathrm{~h}$, PXR-null mouse livers had a greater increase of cleaved caspase-3 in comparison with wild-type mice (Figure 6c). Thus, when PXR is absent, hepatocytes are vulnerable to LPS/ GalN-induced apoptotic injury and the commencement of hepatocyte apoptosis is accelerated.

The relative levels of pro- and anti-apoptotic proteins are the key determinants and regulators of cell survival and death. ${ }^{29,30}$ PXR regulates anti-apoptotic proteins $\mathrm{Bcl}-\mathrm{xL}$ and Bcl-2 in primary hepatocytes ${ }^{15}$ and colon cancer cells. ${ }^{16}$ As $\mathrm{Bcl}-\mathrm{xL}$, but not $\mathrm{Bcl}-2$, has been implicated to have a protective role in LPS/GalN-induced acute hepatic failure model, ${ }^{31,32}$ we hypothesized that a lack of PXR might affect downstream anti-apoptotic proteins in LPS/GalN-induced liver injury. The basal levels of Bcl-xL were slightly decreased due to lack of PXR. LPS/GalN transiently and weakly increased Bcl-xL levels in both genotypes of mice. Overall, the levels of Bcl-xL were lower in PXR-null than wild-type mouse livers (Figure 7a). HO-1 is a stress-responsive enzyme that has been implicated as a protective protein through antiapoptotic, anti-inflammatory, and anti-oxidant actions. ${ }^{33,34}$ HO- 1 is directly regulated by Stat 3 and can protect mice from LPS/GalN-induced apoptotic liver injury. ${ }^{35}$ Western blot analysis revealed that wild-type mouse livers had rapid transient induction of $\mathrm{HO}-1$ protein at $1 \mathrm{~h}$, which did not occur in PXR-null (Figure 7a). HO-1 proteins decreased in both genotypes at the time $(5 \mathrm{~h})$ when stat 3 was highly activated. The results indicate that delayed early activation of Stat3 may not be able to result in sufficient transcription of HO-1. Impaired transcription machinery due to severe liver injury at $5 \mathrm{~h}$ may also partly account for the reduction of HO-1. In addition, the levels of Bax were slightly and transiently induced only in LPS/GalN-treated wild-type mice at $1 \mathrm{~h}$ (Figure 7a). Thus, in the absence of PXR, the hepatic anti-apoptotic pathways are compromised in response to LPS/GalN.

Autophagy promotes survival by removing unwanted cellular substances and by providing nutrients under starvation conditions. ${ }^{36}$ It could, however, also contribute to cell death if the process is deregulated. ${ }^{36}$ The role of autophagy in LPS/ GalN-induced liver injury has not been studied. We found that LPS/GalN-induced LC3B (Figure 7b), a marker of autophagy, ${ }^{37}$ within $1 \mathrm{~h}$ in both genotypes. LC3B-II, which is converted from LC3B-I by lipidation, is located on the autophagic vacuoles. ${ }^{37}$ Five hours after treatment, LPS/GalNinduced expression of LC3B-I and -II was sustained in the livers of wild-type mice. In contrast, LC3B-I and -II proteins of PXR-null mice rapidly dropped to levels lower than basal levels. The levels of Beclin 1, which is a necessary participant in autophagic vacuoles formation and also takes part in trafficking of lysosomal proteins, ${ }^{38,39}$ were reduced by LPS/ GalN treatment in both genotypes (Figure 6b). However, the reduction was transient in wild type but sustained in PXRnull mouse livers. Taken together, our results indicate that autophagy does have a role in LPS/GalN-induced liver injury and that autophagy is likely a defensive mechanism to protect 
a

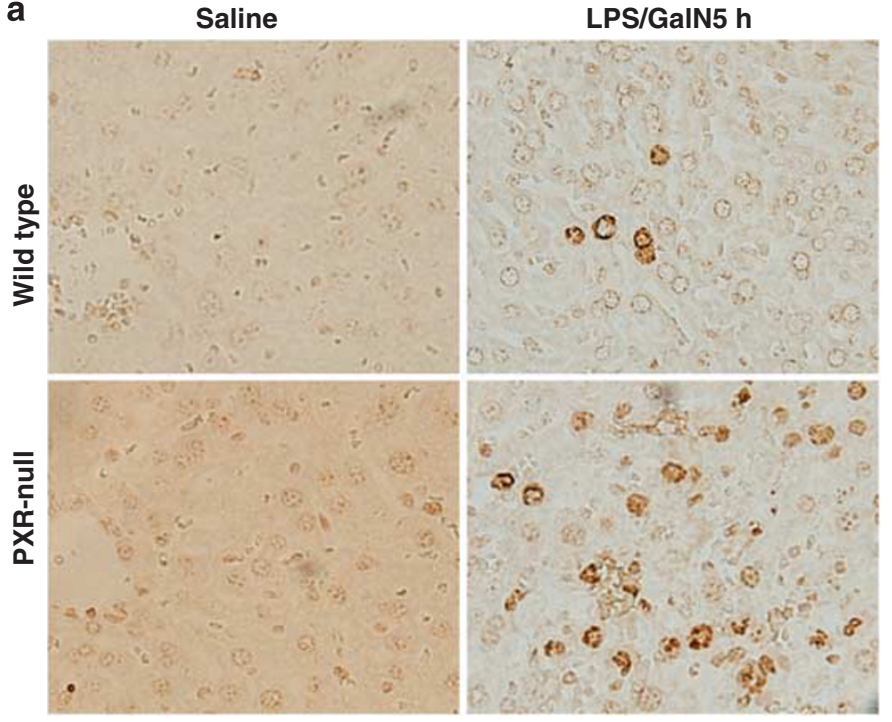

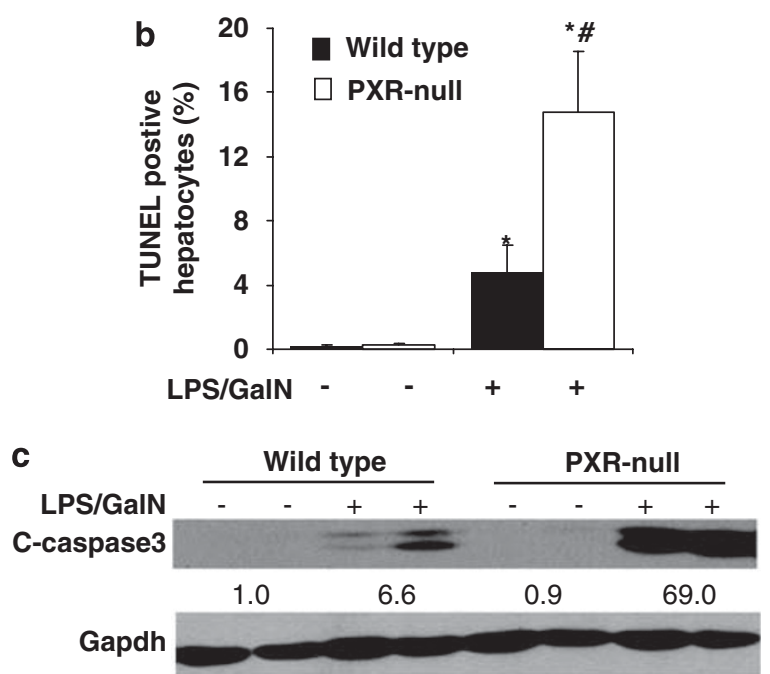

Figure 6 LPS/GalN-induced hepatocyte apoptosis is augmented in PXR-null mice. Mice were i.p. injected with LPS (100 $\mu \mathrm{g} / \mathrm{kg})$ and GalN (700 mg/kg) or saline ( $n=5 /$ group/time point). (a) Representative TUNEL staining of liver sections of wild-type and PXR-null mice i.p. injected with LPS/GalN or saline for $5 \mathrm{~h}$. (b) Quantification of TUNEL-positive hepatocytes of liver sections of wild-type or PXR-null mice $5 \mathrm{~h}$ after LPS/GalN injection. ${ }^{\star} P<0.05$, compared with wild-type mice of $0 \mathrm{~h}$; ${ }^{\#} P<0.05$, compared within same time point. (c) Immunoblot analysis of cleaved caspase-3. Representative samples are shown in the figure.

a
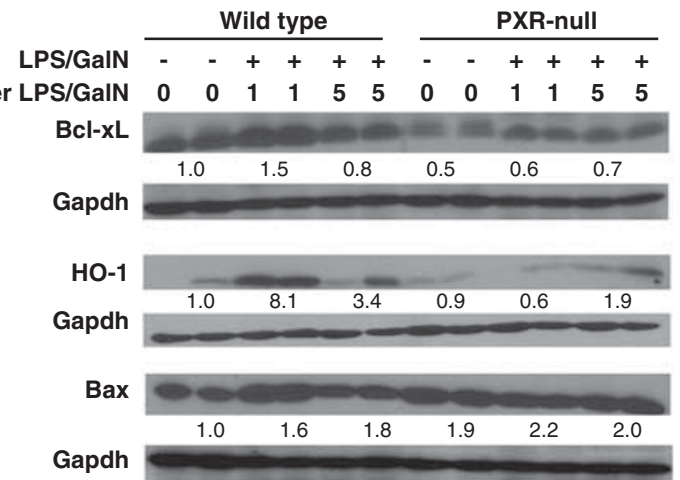

b

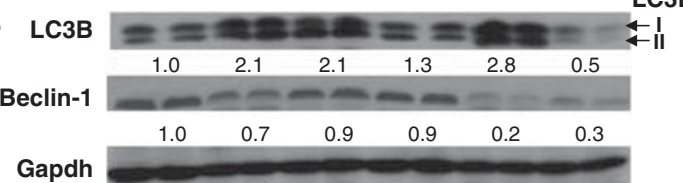

Figure 7 Loss of PXR decreases the levels of anti-apoptotic proteins and alters the expression of proteins involved in autophagy pathways after LPS/GaIN injection. (a) Anti-apoptotic protein data. (b) Autophagy protein data. Mice were i.p. injected with LPS $(100 \mu \mathrm{g} / \mathrm{kg})$ and GalN $(700 \mathrm{mg} / \mathrm{kg})$ or saline ( $n=5 /$ group/time point). Livers were removed at the time point as indicated. Liver proteins were immunoblotted with indicated antibodies and representative samples are shown in the figure.

liver from injury. The hepatoprotective effects of autophagy are compromised due to lack of PXR.

\section{DISCUSSION}

This study focuses on the in vivo role of PXR in LPS/GalNinduced hepatocyte apoptosis and liver injury. The data in- dicate that in response to LPS/GalN, PXR-null mice had increased hepatocyte apoptosis and augmented liver injury, which suggests the protective role of PXR in LPS/GalNinduced acute liver injury. Our findings suggest that (1) PXR is essential for Jak2/Stat3-mediated survival signals; (2) PXR is implicated in MAP kinase pathways and regulates cell survival; and (3) PXR confers anti-apoptotic effects through Bcl-xL, HO-1, and autophagy.

\section{PXR is Implicated in LPS/GaIN-mediated MAP Kinase Pathway}

LPS/GalN treatment caused increased apoptotic, necrotic, and hemorrhagic liver injury in PXR-null mice in comparison with wild-type mice, suggesting that PXR is critical for cell survival. Once bound to TNF $\alpha$, TNFR1 recruits adaptor proteins leading to activation of MAP kinases in the liver. ${ }^{1}$ Earlier studies have shown that JNK inhibitor SP600125 prevents mice from LPS-induced liver injury ${ }^{40}$ and JNK knockout mice are resistant to $\mathrm{TNF} \alpha$-induced liver damage. ${ }^{6,41}$ It is believed that the pro-apoptotic role of JNK1 occurs through activation of E3 ubiquitin ligase Itch and sequential degradation of anti-apoptotic protein $\mathrm{c}-\mathrm{Flip}_{\mathrm{S} / \mathrm{L}}{ }^{41}$ whereas JNK2 directly activates caspase- 8 and promotes apoptosis. ${ }^{6}$ Together with other results, our findings of increased hepatic p-JNK1/2 in PXR-null mice suggest that abrogation of PXR facilitates LPS/GalN-induced JNK activation, which is associated with increased hepatocyte apoptosis. P38 inhibitor SB203580 attenuates LPS-induced liver injury in mice with increased CYP2E1 activity through antioxidative stress mechanisms. ${ }^{40}$ Controversially, hepatocyte p38 $\alpha$-deficient mice have exacerbated JNK activation and 
aggravated LPS-induced hepatic apoptosis and liver injury. ${ }^{11}$ Although the effect is mild, LPS/GalN treatment resulted in a transient reduction of hepatic p-p38. However, reactivation of hepatic p38 was more apparent in PXR-null than wild-type mouse $5 \mathrm{~h}$ after LPS/GalN injection, which coincided with the activation of JNK1/2, ERK1/2, and apoptosis. Activation of ERK1/2 has been proposed to promote cell proliferation and cell cycle of several cell types including cancer cells. On the contrary, accumulating reports show that activation of ERK1/2 is associated with tissue injury including lung, ${ }^{42-44}$ liver, ${ }^{45,46}$ and kidney. ${ }^{47}$ In addition, ERK inhibitor PD98059 attenuates liver injury after trauma-hemorrhage, ${ }^{44}$ indicating that ERK1/2 promotes cell death. Our findings, which show a rapid deactivation of ERK1/2 and lack of p-ERK1/2 in PXRnull livers within $1 \mathrm{~h} \mathrm{LPS} / \mathrm{GalN}$ injection, indicate a prosurvival role of ERK1/2 at early time point. Reactivation of ERK1/2 and higher levels of p-ERK1/2 in PXR-null than in wild-type mice, which coincided with increased p-JNK1/2 as well as cell death and injury at $5 \mathrm{~h}$, suggests a pro-death role of p-ERK1/2 at later time point. Taken together, deregulated phosphorylation of hepatic JNK1/2 and ERK1/2 is found in PXR-null mice. Thus, MAP kinase pathways are implicated in liver injury found in PXR-null mice.

\section{PXR is Implicated in LPS/GaIN-mediated Activation of Stat3}

Stat 3 has an important role in cell survival and carcinogenesis through regulation of proliferation and apoptosis. ${ }^{48}$ Liverspecific inactivation of Stat3 leads to increased hepatocyte apoptosis in mice. ${ }^{4}$ Stat 3 over-expression in rat hepatocytes provides protection against hypoxia/re-oxygenation-induced injury. ${ }^{49}$ Promotion of Stat 3 confers resistance to apoptosis and accelerated proliferation, resulting in enhanced hepatitisinduced hepatocarcinogenesis. ${ }^{50}$ Phosphorylation of Stat3 at Tyr 705 is mediated by Jak family, allowing Stat3 to form dimer and regulate transcription. Phosphorylation of Stat 3 at Ser727 can be regulated by many serine kinases besides Jaks. ERK1/2, mTOR, Vav, Rac-1, and SEK-1/MKK-4 can all phosphorylate Stat3 at Ser727 depending on extracellular stimulus and cell type. De-phosphorylation of Stat3 terminates its activity. In cytoplasm, tyrosine phosphoatases, such as SH-2-containing phosphatase 1 (SHP1), SHP2, and protein-tyrosine phosphatase $1 \mathrm{~B}$ can prevent Stat3 activation. Suppressor of cytokine signaling negatively regulates Stat3 activation. In nucleus, protein inhibitor of activated Stat family blocks Stat-DNA binding. In addition, Stat3 can be de-phosphorylated by nuclear tyrosine phosphatases TC45 that allows it to return to cytoplasm. Recently, it has been uncovered that KAP-1a, a universal co-repressor protein for Kruppel-associated box zinc-finger protein, accelerates de-phosphorylation of Stat3. ${ }^{51}$ Thus, phosphorylation of Stat 3 is a dynamic process and can be complicated. Our data show that LPS/GalN-induced hepatic Stat3 phosporylation at Tyr705 is delayed in PXR-null mice, suggesting that early survival signaling through Stat 3 is compromised in the absence of PXR. Among the three upstream kinases that phosphorylate Stat3, only Jak2 (Tyr1007/1008) early activation is markedly blocked due to PXR deficiency. Phosphorylation of Stat3 at Ser727 was comparable between wild-type and PXR-null mice, thus, PXR does not affect activation of Stat3 at Ser727. Together, our results show a novel function of PXR, that is, PXR positively and preferentially regulates LPS/ GalN-induced phosphorylation of Jak2, which is associated with the protective action of PXR in vivo. Additional study needs to be done to determine how PXR regulates Jak2 activation.

\section{PXR-mediated Anti-apoptotic Effect is Essential for Hepatocyte Survival}

The anti-apoptotic effects of PXR have been observed in primary human or rat hepatocytes through up-regulation of Bcl-xL and Bcl-2. Downregulation of PXR contributes to cell growth inhibition and apoptosis in endometrial cancer cells. The crucial role of $\mathrm{Bcl}-\mathrm{xL}$ in antagonizing Bid-mediated hepatocyte apoptosis is evidenced by the fact that mice with hepatocyte specific-Bcl-xL deficiency develop spontaneous and continuous apoptosis. ${ }^{52} \mathrm{Bcl}-\mathrm{xL}$, but not $\mathrm{Bcl}-2$, has been implicated in the protection of LPS/GalN-induced acute hepatic failure model. ${ }^{31,32,53}$ In line with the above reports, we found that protein levels of $\mathrm{Bcl}-\mathrm{xL}$ were reduced in the livers of untreated as well as LPS/GalN-treated PXR-null mice. Bcl-xL is also one of the Stat 3 target genes, ${ }^{54}$ correspondingly, we observed that the Bcl-xL levels were higher in wild-type than PXR-null mice $1 \mathrm{~h}$ after the treatment. Thus, it is likely that impaired activation of Stat 3 in PXR-null mice may affect expression and function of Bcl-xL. HO-1 system has been shown to protect tissue from injury in liver, ${ }^{35}$ lung, ${ }^{55,56}$ and intestine, ${ }^{57,58}$ after LPS/GalN ${ }^{59}$ or LPS treatment, ${ }^{35}$ ischemia-reperfusion, ${ }^{60}$ and transplantation ${ }^{57,58}$ through anti-apoptosis, anti-oxidant, and anti-inflammation actions. More recently, $\mathrm{HO}-1$ and Stat 3 are implicated in protection against hyperoxia-induced lung injury. ${ }^{61}$ The underlying mechanisms include Stat3-mediated activation of HO-1 and consequential increases of its product, carbon monoxide, which in turn increases Stat3 activation. Induction of HO-1 can activate its downstream survival targets such as pAkt, Bcl-xL, and Bcl-2. ${ }^{61}$ We found that LPS/GalNinduced early increase of $\mathrm{HO}-1$ was markedly reduced in the livers of PXR-null mice, which is well correlated with the decreased pro-survival molecules Bcl-xL and pAkt in PXRnull mice. In addition, downregulation of Bax protects mice from GalN/TNF $\alpha$-induced liver damage. ${ }^{62}$ The fact that LPS/ GalN modestly induced Bax in wild-type mice, but not in PXR-null mice, indicates that PXR might not have a significant role in regulating Bax-mediated pro-apoptosis pathway. Our results point out that PXR positively regulates anti-apoptotic events mainly through Stat3, Bcl-xL, and HO-1. Stat3 modulates multiple downstream pro-survival pathways, of which Bcl-xL and HO-1 are only two; therefore, we could not exclude the possibility that there are additional 
pro-survival molecules regulated by Stat 3 that confer protective effects.

$\mathrm{Bcl}-\mathrm{xL}^{54}$ and $\mathrm{HO}-\mathrm{H}^{61}$ are critical survival genes directly regulated by Stat3. Bcl-xL is also regulated by $\mathrm{PXR},{ }^{15}$ and we consistently observed reduced basal levels of $\mathrm{Bcl}-\mathrm{xL}$ protein in PXR-null mice. We also found LPS/GalN-induced HO-1 is greatly reduced due to lack of PXR. Our data indicate a presence of crosstalk between these two important transcription factors Stat 3 and PXR and that they may or may not target overlapping downstream molecules that eventually confer significant protection against LPS/GalN-induced liver injury. As indicated in our data, sporadic hepatocyte apoptosis and neutrophil infiltration occurred in the livers of PXR-null mice. Neither elevated ALT nor cleaved caspase-3 was detected accordingly, but, slightly elevated basal levels of p-Stat3 (Tyr705), p-p38, and p-Akt as well as decreased basal levels of Bcl-xL were found in livers of PXR-null mice. Therefore, PXR is important in maintaining hepatocyte homeostasis. In the absence of PXR, the balance between prosurvival and pro-apoptosis is shifted. Our novel findings delineate an earlier unacknowledged interdependence between PXR and Stat3 in vivo and help us to better understand the potential usage of PXR as a therapeutic target for acute liver injury.

\section{ACKNOWLEDGEMENTS}

We thank Dr James $\mathrm{P}$ Luyendyk for critical discussion, Mrs Lin He for technical support, and Ms Barbara Brede for editing this manuscript. This work was supported by NIH grants CA53596, AA14147, and COBRE Molecular Biology Core, P20 RR021940.

\section{DISCLOSURE/CONFLICT OF INTEREST}

The authors declare no conflict of interest.

1. Wajant $H$, Pfizenmaier $K$, Scheurich $P$. Tumor necrosis factor signaling. Cell Death Differ 2003;10:45-65.

2. Karin M, Lin A. NF-kappaB at the crossroads of life and death. Nat Immunol 2002;3:221-227.

3. Beg AA, Sha WC, Bronson RT, et al. Embryonic lethality and liver degeneration in mice lacking the RelA component of NF-kappa B. Nature 1995;376:167-170.

4. Streetz KL, Wustefeld T, Klein C, et al. Lack of gp130 expression in hepatocytes promotes liver injury. Gastroenterology 2003;125: 532-543.

5. Levy DE, Darnell Jr JE. Stats: transcriptional control and biological impact. Nat Rev Mol Cell Biol 2002;3:651-662.

6. Wang $\mathrm{Y}$, Singh $\mathrm{R}$, Lefkowitch $\mathrm{JH}$, et al. Tumor necrosis factor-induced toxic liver injury results from JNK2-dependent activation of caspase-8 and the mitochondrial death pathway. J Biol Chem 2006;281: 15258-15267.

7. Zhuang S, Schnellmann RG. A death-promoting role for extracellular signal-regulated kinase. J Pharmacol Exp Ther 2006;319:991-997.

8. He X, Wang J, Guo Z, et al. Requirement for ERK activation in sinomenine-induced apoptosis of macrophages. Immunol Lett 2005;98:91-96.

9. Wang $X$, Martindale JL, Holbrook NJ. Requirement for ERK activation in cisplatin-induced apoptosis. J Biol Chem 2000;275:39435-39443.

10. Roulston A, Reinhard C, Amiri P, et al. Early activation of C-Jun Nterminal kinase and p38 kinase regulate cell survival in response to tumor necrosis factor alpha. J Biol Chem 1998;273:10232-10239.

11. Heinrichsdorff J, Luedde T, Perdiguero E, et al. p38 alpha MAPK inhibits JNK activation and collaborates with IkappaB kinase 2 to prevent endotoxin-induced liver failure. EMBO Rep 2008;9:1048-1054.
12. Zhou C, Tabb MM, Nelson EL, et al. Mutual repression between steroid and xenobiotic receptor and NF-kappaB signaling pathways links xenobiotic metabolism and inflammation. J Clin Invest 2006;116: 2280-2289.

13. Shah $Y M, M a X$, Morimura $K$, et al. Pregnane $X$ receptor activation ameliorates DSS-induced inflammatory bowel disease via inhibition of NF-kappaB target gene expression. Am J Physiol Gastrointest Liver Physiol 2007;292:G1114-G1122.

14. Wang K, Wan YJ. Nuclear receptors and inflammatory diseases. Exp Biol Med (Maywood) 2008;233:496-506.

15. Zucchini N, de Sousa G, Bailly-Maitre $B$, et al. Regulation of BCl-2 and $\mathrm{Bcl}-\mathrm{xL}$ anti-apoptotic protein expression by nuclear receptor PXR in primary cultures of human and rat hepatocytes. Biochim Biophys Acta 2005; 1745:48-58.

16. Zhou J, Liu $M$, Zhai $Y$, et al. The antiapoptotic role of pregnane $X$ receptor in human colon cancer cells. Mol Endocrinol 2008;22:868-880.

17. Haughton EL, Tucker SJ, Marek CJ, et al. Pregnane $\mathrm{X}$ receptor activators inhibit human hepatic stellate cell transdifferentiation in vitro. Gastroenterology 2006;131:194-209.

18. Marek CJ, Tucker SJ, Konstantinou DK, et al. Pregnenolone-16alphacarbonitrile inhibits rodent liver fibrogenesis via PXR (pregnane $X$ receptor)-dependent and PXR-independent mechanisms. Biochem J 2005;387(Part 3):601-608.

19. Langmade SJ, Gale SE, Frolov A, et al. Pregnane $X$ receptor (PXR) activation: a mechanism for neuroprotection in a mouse model of Niemann-Pick C disease. Proc Natl Acad Sci USA 2006;103: 13807-13812.

20. Freudenberg MA, Keppler D, Galanos C. Requirement for lipopolysaccharide-responsive macrophages in galactosamine-induced sensitization to endotoxin. Infect Immun 1986;51:891-895.

21. Luster MI, Germolec DR, Yoshida T, et al. Endotoxin-induced cytokine gene expression and excretion in the liver. Hepatology 1994; 19:480-488.

22. Josephs MD, Bahjat FR, Fukuzuka K, et al. Lipopolysaccharide and D-galactosamine-induced hepatic injury is mediated by TNF-alpha and not by Fas ligand. Am J Physiol Regul Integr Comp Physiol 2000;278:R1196-R1201.

23. Xie W, Barwick JL, Downes $M$, et al. Humanized xenobiotic response in mice expressing nuclear receptor SXR. Nature 2000;406:435-439.

24. Saini SP, Mu Y, Gong H, et al. Dual role of orphan nuclear receptor pregnane $X$ receptor in bilirubin detoxification in mice. Hepatology 2005:41:497-505.

25. Bezzi M, Hasmim M, Bieler $G$, et al. Zoledronate sensitizes endothelial cells to tumor necrosis factor-induced programmed cell death: evidence for the suppression of sustained activation of focal adhesion kinase and protein kinase B/Akt. J Biol Chem 2003;278:43603-43614.

26. Shaik ZP, Fifer EK, Nowak G. Protein kinase B/Akt modulates nephrotoxicant-induced necrosis in renal cells. Am J Physiol Renal Physiol 2007;292:F292-F303.

27. Osawa $Y$, Hannun YA, Proia RL, et al. Roles of AKT and sphingosine kinase in the antiapoptotic effects of bile duct ligation in mouse liver. Hepatology 2005;42:1320-1328.

28. Shuai K, Liu B. Regulation of JAK-STAT signalling in the immune system. Nat Rev Immunol 2003;3:900-911.

29. Yang E, Korsmeyer SJ. Molecular thanatopsis: a discourse on the BCL2 family and cell death. Blood 1996;88:386-401.

30. Reed JC, Zha H, Aime-Sempe C, et al. Structure-function analysis of Bcl2 family proteins regulators of programmed cell death. Adv Exp Med Biol 1996:406:99-112.

31. de la Coste $A$, Fabre $M, M c D o n e l l ~ N$, et al. Differential protective effects of $\mathrm{Bcl}-\mathrm{xL}$ and $\mathrm{BCl}-2$ on apoptotic liver injury in transgenic mice. Am J Physiol 1999;277(3 Part 1):G702-G708.

32. Nakama T, Hirono S, Moriuchi A, et al. Etoposide prevents apoptosis in mouse liver with D-galactosamine/lipopolysaccharide-induced fulminant hepatic failure resulting in reduction of lethality. Hepatology 2001;33:1441-1450.

33. Otterbein LE, Bach FH, Alam J, et al. Carbon monoxide has antiinflammatory effects involving the mitogen-activated protein kinase pathway. Nat Med 2000;6:422-428.

34. Otterbein LE, Soares MP, Yamashita K, et al. Heme oxygenase-1: unleashing the protective properties of heme. Trends Immunol 2003;24:449-455. 
35. Sass G, Soares MC, Yamashita K, et al. Heme oxygenase-1 and its reaction product, carbon monoxide, prevent inflammation-related apoptotic liver damage in mice. Hepatology 2003;38:909-918.

36. Yin XM, Ding WX, Gao W. Autophagy in the liver. Hepatology 2008:47:1773-1785

37. Kabeya Y, Mizushima N, Yamamoto A, et al. LC3, GABARAP and GATE16 localize to autophagosomal membrane depending on form-II formation. J Cell Sci 2004;117(Part 13):2805-2812.

38. Liang $\mathrm{XH}$, Jackson $\mathrm{S}$, Seaman $\mathrm{M}$, et al. Induction of autophagy and inhibition of tumorigenesis by beclin 1. Nature 1999;402:672-676.

39. Kihara A, Kabeya $Y$, Ohsumi $Y$, et al. Beclin-phosphatidylinositol 3-kinase complex functions at the trans-Golgi network. EMBO Rep 2001;2:330-335

40. Wu D, Cederbaum A. Cytochrome P4502E1 sensitizes to tumor necrosis factor alpha-induced liver injury through activation of mitogen-activated protein kinases in mice. Hepatology 2008;47: 1005-1017.

41. Chang L, Kamata H, Solinas G, et al. The E3 ubiquitin ligase itch couples JNK activation to TNFalpha-induced cell death by inducing c-FLIP(L) turnover. Cell 2006;124:601-613.

42. Schuh K, Pahl A. Inhibition of the MAP kinase ERK protects from lipopolysaccharide-induced lung injury. Biochem Pharmacol 2009;77:1827-1834.

43. Bove PF, Hristova M, Wesley UV, et al. Inflammatory levels of nitric oxide inhibit airway epithelial cell migration by inhibition of the kinase ERK1/2 and activation of hypoxia-inducible factor-1 alpha. J Biol Chem 2008:283:17919-17928.

44. Hsu JT, Kan WH, Hsieh $\mathrm{CH}$, et al. Role of extracellular signal-regulated protein kinase (ERK) in 17beta-estradiol-mediated attenuation of lung injury after trauma-hemorrhage. Surgery 2009;145:226-234.

45. Haimerl F, Erhardt A, Sass G, et al. Down-regulation of the deubiquitinating enzyme ubiquitin-specific protease 2 contributes to tumor necrosis factor-alpha-induced hepatocyte survival. J Biol Chem 2009;284:495-504.

46. Zhai $Y$, Shen XD, Gao F, et al. CXCL10 regulates liver innate immune response against ischemia and reperfusion injury. Hepatology 2008:47:207-214.

47. Mkaddem SB, Werts C, Goujon JM, et al. Heat shock protein gp96 interacts with protein phosphatase 5 and controls toll-like receptor 2 (TLR2)-mediated activation of extracellular signal-regulated kinase (ERK) $1 / 2$ in post-hypoxic kidney cells. J Biol Chem 2009;284: 12541-12549.

48. Aggarwal BB, Sethi G, Ahn KS, et al. Targeting signal-transducer-andactivator-of-transcription-3 for prevention and therapy of cancer: modern target but ancient solution. Ann N Y Acad Sci 2006; 1091:151-169.

49. Terui K, Enosawa S, Haga S, et al. Stat3 confers resistance against hypoxia/reoxygenation-induced oxidative injury in hepatocytes through upregulation of Mn-SOD. J Hepatol 2004;41:957-965.

50. Ogata H, Kobayashi T, Chinen T, et al. Deletion of the SOCS3 gene in liver parenchymal cells promotes hepatitis-induced hepatocarcinogenesis. Gastroenterology 2006;131:179-193.

51. Tsuruma R, Ohbayashi N, Kamitani S, et al. Physical and functional interactions between STAT3 and KAP1. Oncogene 2008;27:3054-3059.

52. Takehara T, Tatsumi T, Suzuki T, et al. Hepatocyte-specific disruption of Bcl-xL leads to continuous hepatocyte apoptosis and liver fibrotic responses. Gastroenterology 2004;127:1189-1197.

53. Kosai K, Matsumoto K, Funakoshi $\mathrm{H}$, et al. Hepatocyte growth factor prevents endotoxin-induced lethal hepatic failure in mice. Hepatology 1999:30:151-159.

54. Bromberg JF, Wrzeszczynska MH, Devgan G, et al. Stat3 as an oncogene. Cell 1999:98:295-303.

55. Fredenburgh LE, Baron RM, Carvajal IM, et al. Absence of heme oxygenase-1 expression in the lung parenchyma exacerbates endotoxin-induced acute lung injury and decreases surfactant protein-B levels. Cell Mol Biol (Noisy-le-grand) 2005;51:513-520.

56. Zampetaki A, Minamino T, Mitsialis SA, et al. Effect of heme oxygenase-1 overexpression in two models of lung inflammation. Exp Biol Med (Maywood) 2003;228:442-446.

57. Kaizu T, Ikeda A, Nakao A, et al. Protection of transplant-induced hepatic ischemia/reperfusion injury with carbon monoxide via MEK ERK1/2 pathway downregulation. Am J Physiol Gastrointest Liver Physiol 2008;294:G236-G244.

58. Nakao A, Otterbein LE, Overhaus $M$, et al. Biliverdin protects the functional integrity of a transplanted syngeneic small bowel. Gastroenterology 2004;127:595-606.

59. Tsui TY, Obed A, Siu YT, et al. Carbon monoxide inhalation rescues mice from fulminant hepatitis through improving hepatic energy metabolism. Shock 2007;27:165-171.

60. Zhang $X$, Shan $P$, Jiang $D$, et al. Small interfering RNA targeting heme oxygenase-1 enhances ischemia-reperfusion-induced lung apoptosis. J Biol Chem 2004;279:10677-10684.

61. Zhang $X$, Shan P, Jiang $G$, et al. Endothelial STAT3 is essential for the protective effects of $\mathrm{HO}-1$ in oxidant-induced lung injury. FASEB J 2006;20:2156-2158.

62. Sass G, Shembade ND, Haimerl F, et al. TNF pretreatment interferes with mitochondrial apoptosis in the mouse liver by A20-mediated down-regulation of Bax. J Immunol 2007;179:7042-7049. 